\title{
HyPER: Heurística de deposição de infraestruturas auxiliares para Redes Veiculares
}

\author{
Pedro H. Souza ${ }^{1}$, Massilon L. Fernandes ${ }^{1}$, Thiago S. Gomides ${ }^{1}$, \\ Fernanda S.H. Souza ${ }^{1}$, Cristiano M. Silva ${ }^{2,3}$ e Daniel L. Guidoni ${ }^{1}$ \\ ${ }^{1}$ Departamento de Ciência da Computação (DCOMP/UFSJ) \\ ${ }^{2}$ Departamento de Tecnologia pela UFSJ (DTECH/UFSJ) \\ Universidade Federal de São João Del Rei (UFSJ), MG - Brasil \\ ${ }^{3}$ Departamento de Computação (DECOM/UFOP) \\ Universidade Federal de Ouro Preto (UFOP), MG - Brasil \\ \{pedronhds, massilon, gomides, fsumika, cristiano, guidoni\}@ufsj.edu.br
}

\begin{abstract}
In this work the HyPER algorithm is presented. This algorithm consists of the combination of two combinatorial optimization techniques, GRASP and Path Relinking, to solve the communication infrastructure (RSU) deployment problem using the Deployment $\Delta\left(\begin{array}{l}\rho^{1} \\ \rho^{2}\end{array}\right)$ metric. This metric aims to establish communication guarantees based on the duration of contact between vehicles and the infrastructures. HyPER was compared to a greedy strategy and the results show that HyPER uses a smaller number of RSUs considering the same performance requirements.
\end{abstract}

Resumo. Neste trabalho é apresentado o algoritmo HyPER. Esse algoritmo consiste na combinação de duas técnicas de otimização combinatória, GRASP e Path Relinking, para resolver o problema de distribuição de unidades de comunicação (RSU) utilizando a métrica Deposição $\Delta\left(\begin{array}{c}\rho^{1} \\ \rho^{2}\end{array}\right)$. Essa métrica visa estabelecer garantias de comunicação a partir do tempo de duração do contato entre os veículos e as infraestruturas auxiliares de comunicação. O HyPER foi comparado com uma estratégia gulosa e os resultados mostram que o $\mathrm{Hy}$ PER utiliza um número menor de RSUs considerando os mesmos requisitos de desempenho.

\section{Introdução}

O agrupamento populacional e o desenvolvimento tecnológico são alguns dos fatores responsáveis pelo constante fluxo de migração das pessoas para as grandes cidades [Mabogunje 1970]. Este crescimento em conjunto com a falta de planejamento urbano são elementos prejudiciais ao bem-estar e a qualidade de vida das pessoas, tornando as cidades ambientes mais estressantes e menos produtivos [Ekblad 1993]. Estima-se, segundo [Van Audenhove et al. 2014], que no ano de 2014, 53\% da população mundial estava concentrada nas grandes cidades, devido as melhores oportunidades. No entanto, a previsão é que em 2050 tenhamos um crescimento de 14\%, prejudicando ainda mais a vida da população.

Nesse sentido, com objetivo de minimizar os reflexos da concentração populacional, a incorporação de tecnologias de sensoriamento, análise, controle e comunicação nos centros urbanos têm sido frequentemente discutidas [Bazzan and Klügl 2007]. Essas 
tecnologias, têm por objetivo proporcionar melhorias na infraestrutura urbana e, principalmente, condições que tornem as grandes cidades ambientes mais eficientes e melhores para se viver. Assim, as cidades inteligentes - Smart cities, são alternativas tecnológicas que buscam minimizar os efeitos proporcionados pelo constante fluxo de migração populacional, com menores impactos na rotina das pessoas.

Nesse cenário, populariza-se nos grandes centros, a implantação dos Sistemas Inteligentes de Transporte (STI) que propõe alternativas capazes de promover melhorias na mobilidade urbana. Para isso, os STI, a partir das Redes Veiculares (VANETs), têm como objetivo desenvolver sistemas de gerenciamento de tráfego a partir de redes de comunicação altamente sofisticadas que interagem a partir de suas entidades [Silva et al. 2016], como: veículos, pedestres, sensores de mobilidade, semáforos e infraestruturas auxiliares de comunicação (Roadside Unit - RSU). As VANETs, podem ser classificadas de acordo com o tipo de comunicação utilizada: comunicação entre veículos (V2V), entre veículos e infraestruturas auxiliares (V2I) e o modelo de comunicação híbrido (V2X) que combina as abordagens V2V e V2I.

A comunicação infraestruturada - V2I, proporciona melhorias na conectividade da rede veicular, no entanto, possui um alto custo para sua implantação. Dessa maneira, este trabalho tem como objetivo propor uma técnica de otimização fundamentada no uso de heurísticas, capazes de minimizar a quantidade de infraestruturas necessárias para criar uma rede V2I. Nesse sentido, dado um cenário urbano, nossa proposta HyPER: Heurística de dePosição de infraEstruturas auxiliares para Redes Veiculares, consiste na aplicação de duas técnicas de otimização combinatória, GRASP e Path Relinking, para resolver o problema de deposição de RSUs em Redes Veiculares Infraestruturadas. As seções deste artigo estão organizadas como segue. Os trabalhos relacionados são apresentados na seção 2. A métrica de garantias de comunicação, Deposição $\Delta\left(\begin{array}{l}\rho^{1} \\ \rho^{2}\end{array}\right)$, e a representação da malha rodoviária são expostas nas seções 3 e 4 , respectivamente. A solução proposta é apresentada na seção 5 e a estratégia gulosa $(\mathrm{VpV})$ na seção 6 . A comparação dos algoritmos é apresentada na seção 7 e a conclusão do trabalho é apresentada na seção 8.

\section{Trabalhos Relacionados}

$\mathrm{Na}$ literatura para as redes veiculares, soluções heurísticas têm sido propostas para minimizar os efeitos dos problema de alocação mínima de infraestruturas auxiliares de comunicação [Sarubbi and Silva 2016], [Silva et al. 2016], e [Silva et al. 2017]. Nesse sentido, no desenvolvimento dessas soluções, os autores propõem métricas capazes de proporcionar garantia de comunicação em conjunto a redução do número de RSUs e, consequentemente, a redução do custo de implantação e manutenção da rede de comunicação.

Os autores em [Silva et al. 2016], propõem para a deposição de infraestruturas a métrica denominada Deposição $\Gamma_{D}\left(\begin{array}{c}\tau \\ \rho^{1}\end{array}\right)$ (Gamma Deployment). Nesse sentido, avalia-se a distribuição de infraestruturas de comunicação em relação ao tempo entre contatos dos veículos e as RSUs. Além disso, essa métrica, garante que a porcentagem $\rho^{1}$ de veículos estejam em frequente contato com as RSUs por no máximo $\tau$ segundos de diferença. Segundo os autores, o limite máximo entre contatos permite aos administradores da rede o monitoramento e a detecção de incidentes de trânsito com maior eficiência, além de permitir recomendações das condições do tráfego. 
Em [Sarubbi and Silva 2016], os autores descrevem o problema de deposição de infraestruturas como o Problema de Localização de Máxima Cobertura (PLMC). Para isso, uma heurística gulosa de deposição de RSUs é construída sob o modelo de restrições denominado Deposição $\Delta\left(\begin{array}{c}\rho^{1} \\ \rho^{2}\end{array}\right)$ (Delta-Deployment). Nesse sentido, a deposição delta têm como objetivo garantir que a conexão entre $\rho^{2}$ porcento dos veículos estejam conectados às infraestruturas auxiliares durante uma porcentagem $\rho^{1}$ to tempo total de viagem. Os resultados obtidos indicam que a solução é capaz de diminuir o número de infraestruturas necessárias ao utilizar o tempo de contato relativo entre veículos e infraestrutura. Em [Silva et al. 2017], é proposto uma variação da métrica Delta-Deployment, onde é avaliado múltiplas regiões, permitindo garantir diferentes Qualidade de Serviços (QoS) para cada região.

\section{Deposição $\Delta\left(\begin{array}{l}\rho^{1} \\ \rho^{2}\end{array}\right)$}

Neste trabalho, para avaliar a distribuição das infraestruturas de comunicação, utiliza-se a métrica Deposição $\Delta\left(\begin{array}{c}\rho^{1} \\ \rho^{2}\end{array}\right)$ [Silva and Meira 2015] que, por princípio, estabelece garantias de comunicação a partir do tempo de duração do contato entre os veículos e às infraestruturas auxiliares. Essas garantias se dão por meio dos parâmetros $\rho^{1}$ e $\rho^{2}$. Dessa forma, uma alocação de infraestruturas satisfaz a Deposição $\Delta\left(\begin{array}{c}\rho^{1} \\ \rho^{2}\end{array}\right)$, se $\rho^{2}$ porcento dos veículos tem tempo de contato com as RSUs por no mínimo $\rho^{1}$ porcento dos seus tempos totais de viagem. A apresentação formal da Deposição $\Delta\left(\begin{array}{l}\rho^{1} \\ \rho^{2}\end{array}\right)$ é exposta na Definição 1.

Definição 1: Seja $M$ uma representação de uma malha rodoviária, $V=\left\{v_{1}, v_{2}, \ldots, v_{n}\right\}$ um conjunto dos veículos que viajam em $M$, e $T=\left\{C_{1}, C_{2}, \ldots, C_{n}\right\}$ a trajetória de cada veículo $v_{n} \in V$. Assim, para um determinado veículo $v_{n} \in V$, existe uma trajetória $C_{n} \in T$. Cada $C_{n} \in T$ representa um subconjunto de células urbanas $C_{n}=\left\{u_{1}^{n}, u_{2}^{n}, \ldots, u_{k}^{n}\right\}$ percorridas por um veículo $v_{n}$ durante sua viagem. Seja $V^{\prime} \subset V o$ subconjunto de veículos $v_{n}$ no cenário que permanecem conectados com RSUs durante o tempo $\geq \rho^{1}, \forall u \in C_{n}$. Essa deposição é considerada $\Delta\left(\begin{array}{c}\rho^{1} \\ \rho^{2}\end{array}\right)$ se $\frac{\left|V^{\prime}\right|}{|V|} \geq \rho^{2}$.

\section{Representação da Malha}

Os ambientes urbanos possuem topologias complexas devido aos variados tamanhos e formatos das vias públicas, que são diferentes para cada cidade. Nesse sentido, são numerosas as possibilidades para a alocação das infraestruturas e, consequentemente, torna o problema de deposição de RSUs complexo e de alto custo computacional para resolução. Nesse sentido, para reduzir as regiões candidatas na alocação de infraestruturas, o particionamento da área urbana em um conjunto de $\psi \mathrm{x} \psi$ células urbanas retangulares de mesma dimensão é aplicado ao nosso trabalho. Essa solução é proposta em alguns trabalhos presentes na literatura como [Silva and Meira 2015, Rocha Silva et al. 2016]. Esta simplificação não representa a cobertura de comunicação das RSUs, no entanto, aplicase esta técnica para reduzir as regiões com possibilidades de alocação. Dessa forma, se posicionarmos uma RSU em uma célula $u$, todos os veículos que passarem por $u$ terão cobertura de comunicação. Além disso, o número de células é proporcional à precisão requisitada, assim, para maiores níveis de precisão, aloca-se um maior no número de células urbanas. Como exemplo, as Figuras 1(b) e 1(c) ilustram o particionamento em $8 \times 8 \mathrm{e}$ $20 \times 20$ células urbanas do mapa rodoviário da cidade de Colônia, Alemanha, sob uma região de aproximadamente $400 \mathrm{~km}^{2}$ (Figura 1(a)). Nesse sentido, as trajetórias de cada veículo apresentadas na seção 3 serão definidas por um conjunto de células urbanas. 


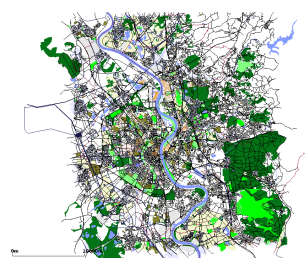

(a) Mapa da cidade

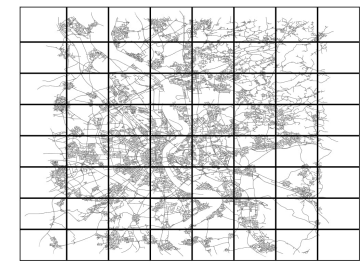

(b) Grid $8 \times 8$

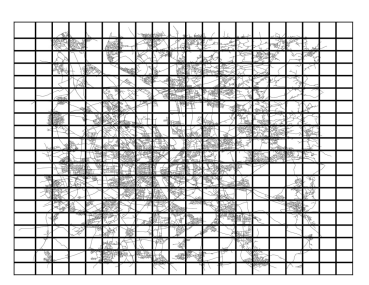

(c) Grid $20 \times 20$

Figura 1. Mapa de células urbanas

\section{HyPER}

Nesta seção apresentamos a estratégia HyPER que, por objetivo, busca promover garantias de comunicação entre veículos e infraestruturas auxiliares em conjunto com a minimização dos custos associados à alocação de cada RSU. Nesse sentido, a heurística apresentada pode ser dividida em dois módulos, sendo esses, GRASP e Path Relinking. No primeiro módulo, para estabelecer garantias de comunicação, a construção de soluções iniciais viáveis é realizada a partir da métrica Deposição $\Delta\left(\begin{array}{c}\rho^{1} \\ \rho^{2}\end{array}\right)$, em seguida, aplica-se busca local para o seu refinamento e, consequentemente, melhora da solução. No segundo módulo, duas das melhores soluções do primeiro módulo são escolhidas e utilizadas para se obter uma outra solução com melhores resultados que as duas utilizadas (Path relinking). Os módulos apresentados são explicados com maiores atenções nas seções 5.1 e 5.2 .

\subsection{GRASP}

O GRASP (Greedy Randomized Adaptive Search Procedure) é um procedimento de busca guloso, adaptativo e aleatório, desenvolvido para a resolução dos problemas de otimização combinatória a partir da construção de um conjunto de soluções promissoras. Nesse sentido, o GRASP constrói suas soluções por meio de um procedimento de execuções independentes, em outras palavras, a cada iteração uma nova solução é gerada. Além disso, a heurística é constituída de duas fases: (5.1.1) Fase de construção e (5.1.2) Busca Local. $\mathrm{Na}$ fase (5.1.1), são construídas soluções iniciais viáveis para o problema. No entanto, não existe garantias de que uma solução que respeita os critérios de viabilidade é uma boa solução. Nesse sentido, é aplicado na etapa (5.1.2) o refinamento das das soluções iniciais. Assim, para cada solução, a busca local cria soluções vizinhas na busca por ótimos locais. Além disso, o número de execuções do GRASP é definido previamente e, as duas fases descritas, são executadas a cada iteração.

\subsubsection{Fase construtiva}

Nesta fase, é realizada a construção de soluções parciais viáveis elemento por elemento considerando a Lista Restrita de Candidatos (RCL). Desta forma, a RCL é uma lista que contém os elementos de melhor benefício que podem ser adicionados à solução em construção. Este é um procedimento construtivo pseudo-guloso controlado pelo parâmetro de aleatoriedade $\alpha$. Nesse sentido, o fator $\alpha$, assume valores no intervalo $0 \leq \alpha \leq 1$, sendo 0 uma escolha totalmente gulosa e 1 uma escolha totalmente aleatória. Destaca-se que, para $(\alpha>0)$, não existe garantias da escolha do melhor elemento, promovendo assim diversificação na construção da solução inicial. Além disso, a RCL é composta dos elementos candidatos ordenados de acordo com os benefícios associados. 


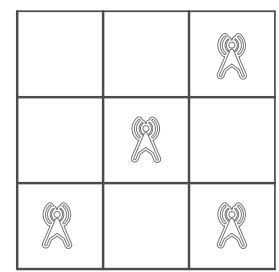

(a) Solução inicial

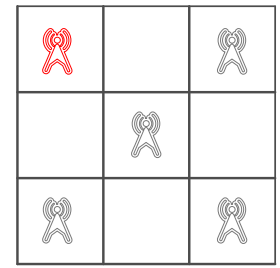

(b) Operação $A D D$

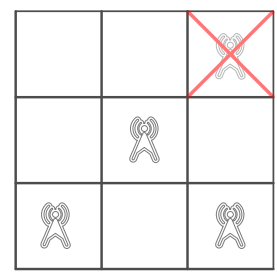

(c) Operação DROP

Figura 2. Vizinhança da Busca local

Neste trabalho, os elementos candidatos são representados pelo conjunto de células urbanas que ainda não possuem RSU. Assim, o benefício associado na incorporação de uma célula à solução está relacionado ao número de veículos cobertos por ela. Dessa forma, quanto maior o número de veículos que passam por uma célula, melhor será o seu benefício para a solução e, quanto maior o tamanho da RCL, maior será o número de possibilidades para construção das soluções.

\subsubsection{Busca Local}

Dada uma solução inicial $S_{0}$, nesta etapa, o GRASP intensifica a exploração da vizinhança $N 1$ de $S_{0}$ em busca dos ótimos locais. Destaca-se que, a vizinhança dessa solução consiste em conjuntos das células urbanas da vizinhança $N 1$ que atendem as restrições determinadas pela Deposição $\Delta\left(\begin{array}{c}\rho^{1} \\ \rho^{2}\end{array}\right)$ [Sarubbi et al. 2017]. Como exemplo, na Figura 2, são apresentadas as operações Add e Drop utilizadas para intensificação da solução inicial. Assim, cada quadrante representa uma célula urbana que pode ou não conter uma RSU. As RSUs em cinza fazem parte da solução inicial depositadas pela fase construtiva, e a RSU em vermelho foi adicionada pela operação $A d d$. Durante essa fase, a solução inicial (figura 2(a)) têm sua vizinhança $N 1$ completamente explorada célula por célula a cada iteração. Além disso, nos casos que uma célula $u$ não possui infraestrutura de comunicação, uma RSU é depositada, operação Add (Figura 2(b)). Por outro lado, se existe uma RSU em $u$, ela é removida a partir da operação Drop (Figura 2(c)).

Após cada operação (Add ou Drop), calcula-se a função objetivo ( $f o$ ) da nova solução e, após o calculo da $f o$, a operação aplicada é revertida. A melhor solução da vizinhança de $S_{0}$ é armazenada devido a estratégia de melhor aprimorante. Além disso, a $f o$ é definida pela minimização do número de RSUs em conjunto com a quantidade de veículos descobertos por essa deposição. Após as operações, para as soluções inviáveis, um fator de penalização é adicionado. Este procedimento, é feito para todas as células urbanas e, para cada operação, o valor da $f o$ e a posição $(x, y)$ dá célula modificada são armazenados. Ao fim das iterações, a operação que proporcionou os melhores ganhos para $f_{o}$, é realizada de fato. Além disso, este processo é repetido até que o critério de parada seja atingido, ou seja, não exista mais melhoras para $f o$.

\subsection{Path Relinking}

O Path-relinking é uma estratégia de intensificação de busca que aprimora as soluções provenientes das etapas de busca local. Para isso, o Path Relinking utiliza duas soluções, $S_{1}$ e $S_{2}$, denominadas solução fonte e solução guia. O objetivo é partir da solução fonte e chegar na solução guia por meio da adição de atributos da solução guia à solução fonte, onde a solução guia possui um resultado melhor que a fonte. A figura 3 exemplifica como é o caminho feito pelo Path Relinking. Primeiro são tomadas duas soluções, a solução 
fonte, em verde, e a solução guia, em vermelho. A partir da solução fonte, são geradas algumas possíveis soluções, em azul. Essas possibilidades são geradas através da adição de atributos da solução guia à solução fonte. Uma possibilidade é adicionar apenas 1 atributo à solução fonte, ou seja, para cada elemento diferente da solução guia aplicado à solução fonte é criada uma possibilidade diferente. As soluções apresentadas neste artigo são matrizes com RSUs depositadas em suas células, e a adição de atributos da solução guia se dá por meio da adição ou retirada de RSUs em determinadas células urbanas da solução fonte. Todos esses candidatos gerados a partir da solução fonte são avaliados. O melhor é então selecionado, em rosa, e este se torna a nova solução fonte. Todo esse procedimento é repetido até que um critério de parada ocorra. Um possível critério de parada é a solução fonte se tornar igual a solução guia. Outro eventual critério de parada é caso as soluções geradas na iteração $i$ não forem melhores que a melhor solução da iteração $i-1$. A possibilidade de gerar soluções com melhores resultados que as duas soluções escolhidas, fonte e guia, é que torna interessante a aplicação desse algoritmo.

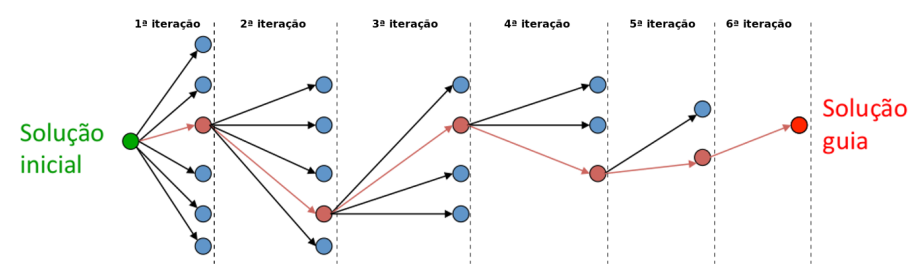

Figura 3. Caminho da solução fonte à solução guia.

\section{Algoritmo base: $\mathrm{VpV}$ - Veículo por Veículo}

A ideia por trás da solução $\mathrm{VpV}$ consiste em iterativamente, de acordo com os parâmetros de entrada requeridos pela Deposição $\Delta\left(\begin{array}{c}\rho^{1} \\ \rho^{2}\end{array}\right)$, avaliar cada veículo separadamente e adicionar RSUs para cobrir o veículo em questão. Um ponto que se deve observar é que as RSUs utilizadas para um veículo atingir a cobertura miníma podem ser suficientes para outros veículos também ficarem cobertos pela deposição. Desse modo, após garantir a cobertura para um determinado veículo, os demais veículos são verificados com base nas novas RSUs adicionadas. O algoritmo termina quando é atingido a garantia miníma requerida pela deposição.

\section{Análise de Resultados}

Nesta seção, a comparação entre o HyPER e o algoritmo VpV é apresentada. Para isso, foi escolhido o trace (rastro) realístico da cidade de Colônia, Alemanha, contendo 75.515 veículos sob um trecho viário de $702 \mathrm{~km}^{2}$. A mobilidade dos veículos, foi simulada a partir da ferramenta SUMO $0.30^{1}$, bem como a localização de cada veículo durante todo o seu percurso. Esta localização, é obtida considerando as coordenadas cartesianas e, convertendo-as em células urbanas. Além disso, o cenário de Colônia foi particionado em um conjunto de $100 \times 100$ células urbanas, assim como apresentado nos trabalhos da seção 2, resultando em células de $270 \mathrm{~m} \times 260 \mathrm{~m}$.

$\mathrm{O}$ desempenho do Hyper é avaliado em relação à estratégia $\mathrm{VpV}$, considerando a minimização do número de infraestruturas auxiliares que mantenham as garantias de comunicação definidas por $\Delta\left(\begin{array}{c}\rho^{1} \\ \rho^{2}\end{array}\right)$. As duas estratégias foram comparadas em relação ao

\footnotetext{
${ }^{1}$ http://sumo.dlr.de/
} 


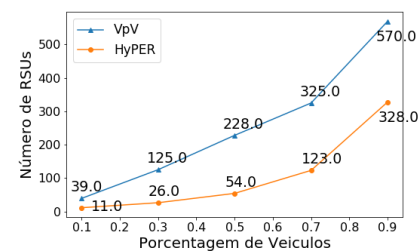

(a) $\rho^{1}=10 \%$

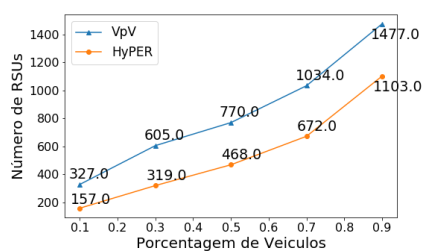

(b) $\rho^{1}=50 \%$

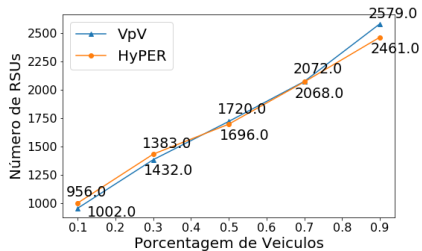

(c) $\rho^{1}=90 \%$

Figura 4. O número de RSUs depositadas por HyPER e VpV. O eixo $x$ representa a quantidade de veículos $\rho^{2}$, enquanto que o eixo $y$ indica o número de infraestruturas alocadas.

número de RSUs que satisfaçam os parâmetros $\rho^{1}$, referente à porcentagem do tempo de viagem que os veículos devem permanecer conectados; e $\rho^{2}$, a porcentagem de veículos que devem estar conectados durante um intervalo maior ou igual a $\rho^{1}$. Para avaliar o comportamento dos algoritmos, considera-se a variação de $\rho^{1}$ e $\rho^{2}$ para os valores $\rho^{1}$ : $\{0.1,0.5$ e 0.9$\}$ e $\rho^{2}:\{0.1,0.3,0.5,0.7$ e 0.9$\}$. No módulo 5.1, GRASP, foram executadas 10 iterações e, consequentemente, dez soluções considerando a Deposição $\Delta\left(\begin{array}{c}\rho^{1} \\ \rho^{2}\end{array}\right)$. As cinco melhores soluções foram selecionadas para aplicarmos a intensificação da busca Path Relinking, em uma combinação simples $C_{5,2}$, ou seja, dez soluções para o problema.

A figura 4 apresenta os resultados após a execução das duas fases do HyPER e da solução VpV. As figuras 4(a), 4(b) e 4(c) mostram os resultados para o conjunto de parâmetros $\rho^{1}$ igual à 10\%, 50\% e 90\%, respectivamente. Para a figura 4(a), de tempo de contato igual à $10 \%$ durante as viagens, observamos que a nossa solução reduz consideravelmente o número de infraestruturas depositadas. Assim, para $\Delta\left(\begin{array}{l}0.1 \\ 0.1\end{array}\right)$ e $\Delta\left(\begin{array}{l}0.1 \\ 0.9\end{array}\right)$, o HyPER alocou, respectivamente, $71 \%$ e $42 \%$ menos RSUs que VpV. Além disso, ao considerarmos um tempo de contato de $\rho^{1}=50 \%$, o HyPER para $\Delta\left(\begin{array}{l}0.5 \\ 0.1\end{array}\right)$, posiciona $52 \%$ menos infraestruturas comparado ao $\mathrm{VpV}$ e para $\Delta\left(\begin{array}{l}0.5 \\ 0.9\end{array}\right)$ posiciona $25 \%$ a menos também comparado ao $\mathrm{VpV}$. Além disso, quando o cenário exige elevados tempos de contato, como para $\rho^{1}=90 \%$, o HyPER aumenta em $4 \%$ considerando $\Delta\left(\begin{array}{l}0.9 \\ 0.1\end{array}\right)$ e, proporciona redução em $4 \%$ ao avaliarmos $\Delta\left(\begin{array}{l}0.9 \\ 0.9\end{array}\right)$, ambos em relação ao $\mathrm{VpV}$. Em ambientes que exigem elevados tempos de contato como $\Delta\left(\begin{array}{l}0.9 \\ 0.9\end{array}\right)$, considera-se quase por completo o percurso dos veículos o que reduz as possibilidades para células senão quase o percurso inteiro dos veículos. O desempenho do HyPER em relação ao $\mathrm{VpV}$ é proveniente das otimizações combinatórias que o HyPER utiliza. A busca local do GRASP adiciona RSUs em células que maximizam o tempo de contato dos veículos e retira das células de baixo impacto para a cobertura do conjunto de veículos. Já o Path Relinking intensifica os ótimos locais encontrados pelo método GRASP por meio da incorporação de atributos de uma solução encontrada em outra, podendo então encontrar melhores soluções.

\section{Conclusões}

Neste trabalho foi apresentada a estratégia HyPER, que consiste na combinação de duas técnicas de otimização combinatória, GRASP e Path Relinking para resolver o problema de minimização da quantidade de RSUs para atender a métrica de Deposição $\Delta\left(\begin{array}{c}\rho^{1} \\ \rho^{2}\end{array}\right)$. Para comparar com o HyPER, foi utilizada a estratégia $\mathrm{VpV}$, explicada na seção 6 . Como apresentada na seção 7, nossa estratégia realiza a deposição de menos RSUs na maioria dos cenários em comparação com VpV para as instâncias apresentadas. Esse resultado é explicado devido ao modo como o HyPER funciona. A busca local do GRASP pro- 
cura sempre encontrar ótimos locais ao procurar pela vizinhança de determinada solução viável, e o Path Relinking visa sempre procurar encontrar outros ótimos locais mudando o ponto de busca através da combinação de atributos de duas soluções que já são ótimos locais. Como trabalhos futuros, pretendemos avaliar a solução utilizando outros traces e cenários.

\section{Agradecimentos}

Os autores gostariam de agradecer a Fundação de Amparo à Pesquisa do Estado de Minas Gerais (FAPEMIG) sob concessão No.: APQ-03120-17 e a Conselho Nacional de Desenvolvimento Científico e Tecnológico (CNPq) sob concessão No.: 150545/2018-5. O presente trabalho foi realizado com apoio da Coordenação de Aperfeiçoamento de Pessoal de Nível Superior - Brasil (CAPES) - Código de Financiamento 001.

\section{Referências}

Bazzan, A. L. and Klügl, F. (2007). Sistemas inteligentes de transporte e tráfego: uma abordagem de tecnologia da informação. Anais das Jornadas de Atualização em Informática, pages 2296-2337.

Ekblad, S. (1993). Stressful environments and their effects on quality ot life in third world cities. Environment and Urbanization, 5(2):125-134.

Mabogunje, A. L. (1970). Systems approach to a theory of rural-urban migration. Geographical Analysis, 2(1):1-18.

Rocha Silva, T., Sarubbi, J., Martins, F., and Silva, C. (2016). Algoritmos baseados na metaheurística grasp para implantação de unidades de comunicação em redes veiculares garantindo qualidade de serviço. XLVIII Simposio Brasileiro de Pesquisa Operacional (SBPO), At Vitória, Espiríto Santo, Brazil.

Sarubbi, J. F., Silva, T. R., Martins, F. V., Wanner, E. F., and Silva, C. M. (2017). A grasp based heuristic for deployment roadside units in vanets. In 2017 IFIP/IEEE Symposium on Integrated Network and Service Management (IM), pages 369-376. IEEE.

Sarubbi, J. F. M. and Silva, C. M. (2016). Delta-r: A novel and more economic strategy for allocating the roadside infrastructure in vehicular networks with guaranteed levels of performance. In Network Operations and Management Symposium (NOMS), 2016 IEEE/IFIP, pages 665-671. IEEE.

Silva, C. M. and Meira, W. (2015). Design of roadside communication infrastructure with qos guarantees. In 2015 IEEE Symposium on Computers and Communication (ISCC), pages 439-444. IEEE.

Silva, C. M., Pitangui, C. G., Guidoni, D. L., Souza, F. S., and Sarubbi, J. F. (2016). Deposição gamma: Alocando infraestrutura de comunicação para redes veiculares garantindo o intervalo" entre contatos" de veículos com a infraestrutura de comunicação. XXXIV Simpósio Brasileiro de Redes de Computadores e Sistemas Distribuídos $S B R C$.

Silva, C. M., Silva, F. A., Sarubbi, J. F., Oliveira, T. R., Meira Jr, W., and Nogueira, J. M. S. (2017). Designing mobile content delivery networks for the internet of vehicles. Vehicular communications, 8:45-55.

Van Audenhove, F.-J., Korniichuk, O., Dauby, L., and Pourbaix, J. (2014). The future of urban mobility 2.0: Imperatives to shape extended mobility ecosystems of tomorrow. 